\title{
离子对型 $[\mathrm{O}, \mathrm{N}, \mathrm{N}]$ 三齿席夫碱镱氯化物的合成及其晶体结构
}

\author{
李邦玉 ${ }^{*} a$ 喻龙宝 ${ }^{b}$ 王耀荣 ${ }^{c}$ 姚英明 $c$ 张 勇 ${ }^{c}$ 沈 琪 ${ }^{c}$ \\ ( ${ }^{a}$ 苏州市职业大学应用化学研究室 苏州 215104) \\ ( ${ }^{b}$ 江苏大学化学化工学院 镇江 212013) \\ (c苏州大学材料与化学化工学部 苏州 215123)
}

\begin{abstract}
摘要 无水 $\mathrm{YbCl}_{3}$ 和席夫碱 $\left[\mathrm{HL}=3,5-(t-\mathrm{Bu})_{2}-2-\mathrm{HOC}_{6} \mathrm{H}_{2} \mathrm{CH}=\mathrm{N}-8-\mathrm{C}_{9} \mathrm{H}_{6} \mathrm{~N}\right]$ 锂盐按 $1: 1$ 物质的量比在 THF 中反应, 得到 离子对席夫碱镜氯化物 $\mathrm{YbLCl}_{3} \mathrm{Li}(\mathrm{DME})_{3}$ (1). 标题配合物 $\mathbf{1}$ 经元素分析、IR 光谱和 $\mathrm{X}$ 射线衍射结构表征. 配合物 $\mathbf{1}$ 属 单斜晶系, $P 2_{1} / n$ 空间群, 晶胞参数: $a=1.4855(16) \mathrm{nm}, b=1.3696(13) \mathrm{nm}, c=2.1816(2) \mathrm{nm}, \alpha=90.00^{\circ}, \beta=98.217(3)^{\circ}, \gamma=$ $90.00^{\circ}, V=4.3748(8) \mathrm{nm}^{3}, Z=4, F(000)=1868, M_{\mathrm{r}}=916.17, D_{\mathrm{c}}=1.397 \mathrm{~g} \cdot \mathrm{cm}^{-3}, \mu=2.364 \mathrm{~mm}^{-1}, R_{1}=0.0855, w R_{2}=0.1863$. 关键词 镱; 席夫碱; 氯化物; 合成; 晶体结构; 离子对
\end{abstract}

\section{Synthesis and Structure of Ion-Pairs Ytterbium Chloride Bearing Tridentate $[\mathrm{O}, \mathrm{N}, \mathrm{N}]$ Schiff-Base Ligand}

\author{
Li, Bangyu $^{*, a} \quad$ Yu, Longbao $^{b} \quad$ Wang, Yaorong $^{c} \quad$ Yao, Yingming $^{c}$ \\ Zhang, Yong ${ }^{c} \quad$ Shen, $\mathrm{Qi}^{c}$ \\ ( ${ }^{a}$ Institute of Applied Chemistry, Suzhou Vocational University, Suzhou 215104) \\ $\left({ }^{b}\right.$ Chemistry and Chemical Engineering, Jiangsu University, Zhenjiang 212013) \\ ( ${ }^{c}$ Chemical Engineering and Materials Science, Suzhou University, Suzhou 215123)
}

\begin{abstract}
The treatment of Schiff base $\mathrm{HL}\left[\mathrm{HL}=3,5-(t-\mathrm{Bu})_{2}-2-\mathrm{HOC}_{6} \mathrm{H}_{2} \mathrm{CH}=\mathrm{N}-8-\mathrm{C}_{9} \mathrm{H}_{6} \mathrm{~N}\right]$ with 1 equiv. $n$-BuLi in THF followed by reacting with 1 equiv. $\mathrm{YbCl}_{3}$ gives the ion-pairs ytterbium Schiff base complex $\mathrm{YbLCl}_{3} \mathrm{Li}(\mathrm{DME})_{3}(\mathbf{1})$. The complex 1 was characterized by elemental analysis, IR spectra, and X-ray structural determination. The crystal data of complex 1 are monoclinic, $P 2_{1} / n$ space group, $a=1.4855(16) \mathrm{nm}, b=1.3696(13) \mathrm{nm}, c=2.1816(2) \mathrm{nm}, \alpha=90.00^{\circ}, \beta=98.217(3)^{\circ}, \gamma=$ $90.00^{\circ}, V=4.3748(8) \mathrm{nm}^{3}, Z=4, F(000)=1868, M_{\mathrm{r}}=916.17, D_{\mathrm{c}}=1.397 \mathrm{~g} \cdot \mathrm{cm}^{-3}, \mu=2.364 \mathrm{~mm}^{-1}, R_{1}=0.0855, w R_{2}=0.1863$.
\end{abstract} Keywords ytterbium; Schiff-base; chloride; synthesis; crystal structure; ion-pairs

近年来，非水体系中席夫碱稀土配合物的合成和催 化性能研究受到关注, 因为在非水体系中, 席夫碱稀土 配合物表现出丰富的反应性能. 2002 年, Piers 等 ${ }^{[1]}$ 用三 烷基稀土配合物 $\mathrm{Ln}\left(\mathrm{CH}_{2} \mathrm{SiMe}_{2} \mathrm{R}\right)_{3}(\mathrm{THF})_{2} \quad(\mathrm{Ln}=\mathrm{Sc}, \mathrm{Y}$; $\mathrm{R}=\mathrm{CH}_{3}, \mathrm{Ph}$ ) 为前体, 合成了一系列二齿席夫碱 3,5$(t-\mathrm{Bu})_{2}-2-\mathrm{HOC}_{6} \mathrm{H}_{2} \mathrm{CH}=\mathrm{N}-2,6-(i-\mathrm{Pr})_{2} \mathrm{C}_{6} \mathrm{H}_{3}$ 的鈧、钎配合物, 并发现席夫碱鈧烷基配合物可以催化胺基炔、烯分子内 氢胺化环化 ${ }^{[2]} .1999$ 年, Evans 等 ${ }^{[3,4]}$ 用四齿乙二胺双水杨 醛席夫碱钾盐与三氯化钎反应生成的席夫碱钎一氯化 物为前体，衍生出了其芳氧化物、硅胺化物、茂化物、
取代乙酰丙酮化物. 文献中，三齿席夫碱稀土配合物的 合成没有二、四齿那么系统. 1990 年, Blech 等 ${ }^{[5]}$ 用 $\mathrm{LaCl}_{3}$ 分别与水杨醛苯胺席夫碱 $2-\mathrm{HOC}_{6} \mathrm{H}_{4} \mathrm{CH}=\mathrm{N}-2,4,6-$ $\mathrm{Me}_{3} \mathrm{C}_{6} \mathrm{H}_{2}$ 的钠盐、乙二胺单水杨醛 $2-\mathrm{HOC}_{6} \mathrm{H}_{4} \mathrm{CH}=$ $\mathrm{N}\left(\mathrm{CH}_{2}\right)_{2} \mathrm{NMe}_{2}$ 的钠盐反应，得到席夫碱镧一氯化物. 钱 延龙等 ${ }^{[6]}$ 合成了水杨醛烷氧基苯胺席夫碱茂化物. 近年 来, 我们采用喹啉胺水杨醛席夫碱 $3,5-(t-\mathrm{Bu})_{2}-$ $2-\mathrm{HOC}_{6} \mathrm{H}_{2} \mathrm{CH}=\mathrm{N}-8-\mathrm{C}_{9} \mathrm{H}_{6} \mathrm{~N}$ (HL) 为辅助配体, 合成了一 系列三齿席夫碱稀土配合物，包括席夫碱稀土一氯化 物、席夫碱稀土二氯化物、均配型席夫碱稀土配合物、

\footnotetext{
*E-mail: 1by@jjssvc.edu.cn; qshen@suda.edu.cn

Received May 29, 2011; revised September 12, 2011; accepted November 1, 2011.

Project supported by the Ph.D Programs Foundation (No. 2009BS01), the Youth Innovation Foundation (No. 2010SZDY12) and the College Students Innovation Foundation (No. 2010SZDX11) of Suzhou Vocational University.

苏州市职业大学博士基金(No. 2009BS01)、校创新基金(No. 2010SZDYY12)和校大学生创新基金(No. 2010SZDX11)资助项目.
} 
席夫碱稀土茂化物、席夫碱稀土胺化物和席夫碱稀土芳 氧化物等, 探讨了部分配合物的催化聚合性能. 其中, Scheme 1 所示为席夫碱镱的系列配合物 ${ }^{[7 \sim 13]}$. Scheme 1 中的大部分内容都已发表, 本文继续报道了以该席夫碱 为辅助配体的离子对席夫碱镱氯化物的合成及其晶体 结构.

\section{1 实验部分}

本研究所描述的实验均采用 Schlenk 实验技术, 在 氩气保护和严格的无水无氧条件下进行.

\section{1 试剂和分析}

所用溶剂使用前均在氩气保护下, 经 $\mathrm{Na}$-二苯甲酮 回流蒸馏. 测试仪器为: Electrochemical Engineering Limited 8100 数字熔点仪(温度计未校正); Carlo-Erba 公 司生产的 EA-1110 型元素分析仪; Hitachi 180-80 polarized Zooman 型原子吸收光谱仪; Rigaku Mercury 型单晶 四圆衍射仪; Magna-550 型红外光谱仪, $\mathrm{KBr}$ 压片. 席夫 碱 $\mathrm{HL}\left[\mathrm{HL}=3,5-(t-\mathrm{Bu})_{2}-2-\mathrm{HOC}_{6} \mathrm{H}_{2} \mathrm{CH}=\mathrm{N}-8-\mathrm{C}_{9} \mathrm{H}_{6} \mathrm{~N}\right]$ 按文 献[14]方法制备.

\section{2 $\mathrm{YbLCl}_{3} \mathrm{Li}(\mathrm{DME})_{3}(1)$ 的合成}

在一 $78{ }^{\circ} \mathrm{C}$ 下, 将 $5.01 \mathrm{~mL}, 1.0 \mathrm{~mol} / \mathrm{L}$ 的 BuLi (5.01 $\mathrm{mmol})$ 己烷溶液用针筒滴加到席夫碱 HL (1.80 g, 5.01 $\mathrm{mmol}$ 的 THF 溶液中, 混合物搅拌 $2 \mathrm{~h}$, 逐渐升至室温, 再把上述深红色溶液滴加到悬浮的 $\mathrm{YbCl}_{3}(1.40 \mathrm{~g}, 5.00$ mmol) THF (20 mL)体系中, 室温搅拌反应过夜, 减压 除去挥发性溶剂, 用热甲苯萃取 2 3 次, 合并、浓缩萃
取液， $-5{ }^{\circ} \mathrm{C}$ 低温下放置一周，析出黄色棒状晶体 3.18 克, 产率 $69.5 \%$, 熔点 $246 \sim 249{ }^{\circ} \mathrm{C}$ (分解). 适于 $\mathrm{X}$ 射线 晶体衍射的单晶在 THF 和 DME 混合溶液中培养得到. IR (KBr pellet) v: 2956 (s), 2928 (s), 2866 (s), 1611 (s), 1534 (m), 1503 (m), 1463 (m), 1320 (m), 1234 (s), 1161 (s), 1086 (m), 834 (m), 784 (m), 635 (m), 558 (m), 508 (m) $\mathrm{cm}^{-1}$. Anal. calcd for $\mathrm{C}_{36} \mathrm{H}_{57} \mathrm{Cl}_{3} \mathrm{~N}_{2} \mathrm{O}_{7} \mathrm{YbLi}$ : C 47.25, $\mathrm{H}$ 6.32, N 3.09, $\mathrm{Cl} 11.62$, Yb 18.81, Li 0.76, found $\mathrm{C} 47.35$, H 6.37, N 3.18, Cl 11.50, Yb 18.77, Li 0.67.

\section{3 配合物晶体结构的测定}

在氩气保护下，挑选适当的晶体装入毛细管中，封 管，低温[193(2) K]下，于 Rigaku Mercury 单晶衍射仪上 用石墨单色化的 Mo K $\alpha$ 射线 $(\lambda=0.071070 \mathrm{~nm})$ 收集数据, 采用 $\varphi / \omega$ 扫描方式, 在 $6.24^{\circ} \leqslant 2 \theta \leqslant 54.96^{\circ}$ 范围内收集衍 射点, 全部数据经过 multi-scan 吸收校正, 结构采用 SHELEXL-97 程序解析, 全矩阵最小二乘法修正. 表 1 给出了配合物 $\mathbf{1}$ 的晶体学数据. 数据存于英国剑桥数据 中心, CCDC 为 817794 .

\section{2 结果与讨论}

\section{1 配合物的合成}

在非水溶剂中, 采用 $\mathrm{LnCl}_{3}$ 和席夫碱碱金属盐的复 分解反应，是制备席夫碱稀土氯化物的常用方法 ${ }^{[3]}$. 我 们前期曾报道了用 $\mathrm{YbCl}_{3}$ 和席夫碱钠盐 $[\mathrm{NaL}(\mathrm{THF})]_{2}$ $\left[\mathrm{HL}=3,5-(t-\mathrm{Bu})_{2}-2-\mathrm{HOC}_{6} \mathrm{H}_{2} \mathrm{CH}=\mathrm{N}-8-\mathrm{C}_{9} \mathrm{H}_{6} \mathrm{~N}\right]$ 来制备席 夫碱镱氯化物 $\left[\mathrm{YbLCl}_{2}(\mathrm{DME})\right]^{[9]},\left[\mathrm{YbL}_{2} \mathrm{Cl}(\mathrm{THF})_{3.5}\right]^{[13]} . \mathrm{X}$

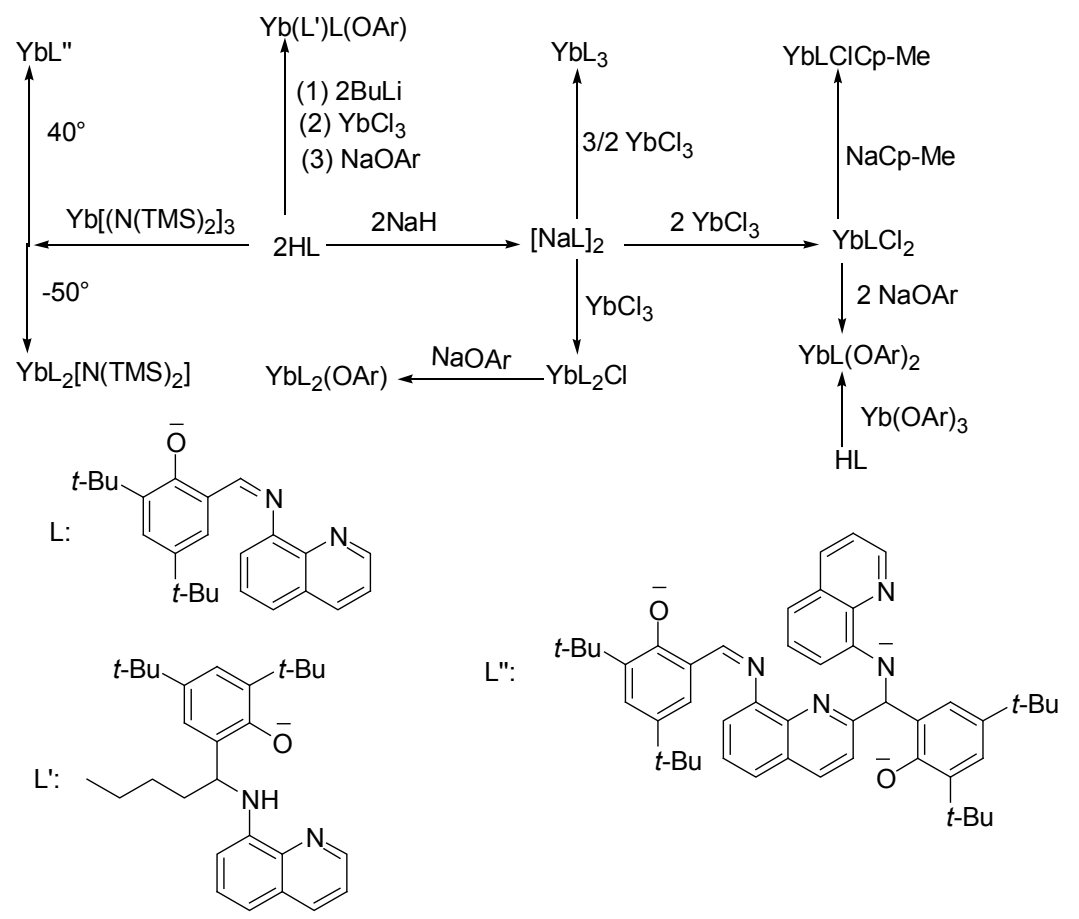

Scheme 1 
表 1 配合物 1 的晶体学数据

Table 1 Crystallographic data and refinements for complex 1

\begin{tabular}{|c|c|}
\hline Empirical formula & $\mathrm{C}_{36} \mathrm{H}_{57} \mathrm{Cl}_{3} \mathrm{~N}_{2} \mathrm{O}_{7} \mathrm{YbLi}$ \\
\hline Formula weight & 916.17 \\
\hline$T / \mathrm{K}$ & $193(2)$ \\
\hline Radiation & Mo K $\alpha$ \\
\hline$\lambda / \mathrm{nm}$ & 0.071070 \\
\hline Crystal system & Monoclinic \\
\hline Space group & $P 2_{1} / n$ \\
\hline$a / \mathrm{nm}$ & $1.48550(16)$ \\
\hline$b / \mathrm{nm}$ & $1.36396(13)$ \\
\hline$c / \mathrm{nm}$ & $2.1816(2)$ \\
\hline$\alpha /\left({ }^{\circ}\right)$ & 90 \\
\hline$\beta /\left(^{\circ}\right)$ & $98.217(3)$ \\
\hline$\gamma /\left(^{\circ}\right)$ & 90 \\
\hline$V / \mathrm{nm}^{3}$ & $4.3748(8)$ \\
\hline$Z$ & 4 \\
\hline$D_{\mathrm{c}} /\left(\mathrm{g} \cdot \mathrm{cm}^{-3}\right)$ & 1.391 \\
\hline$\mu(\mathrm{Mo} \mathrm{K} \alpha) / \mathrm{mm}^{-1}$ & 2.364 \\
\hline$F(000)$ & 1868 \\
\hline Crastal size $/ \mathrm{mm}^{3}$ & $0.40 \times 0.34 \times 0.12$ \\
\hline$\theta$ range for data collection $/\left(^{\circ}\right)$ & 3.12 to 27.48 \\
\hline Limiting indices & $\begin{array}{l}-19 \leqslant h \leqslant 19,-16 \leqslant k \leqslant \\
17,-28 \leqslant l \leqslant 24\end{array}$ \\
\hline Reflections collected/unique & $48060 / 10013\left[R_{\mathrm{int}}=0.0450\right]$ \\
\hline Completeness to $\theta=27.48^{\circ}$ & $99.8 \%$ \\
\hline Max. and min. transmission & 0.7646 and 0.4515 \\
\hline Data/restraints/parameters & $10013 / 19 / 384$ \\
\hline Goodness of fit on $F^{2}$ & 1.140 \\
\hline Refinement method & $\begin{array}{l}\text { Full-matrix least-squares on } \\
F^{2}\end{array}$ \\
\hline Final $R$ indices $[I>2 \sigma(I)]$ & $R_{1}=0.0855, w R_{2}=0.1863$ \\
\hline$R$ indices (all data) & $R_{1}=0.0950, w R_{2}=0.1918$ \\
\hline \multicolumn{2}{|c|}{ Largest diff. peak and hole $/\left(\mathrm{e}^{\circ} \mathrm{nm}^{-3}\right) 2034$ and -1860} \\
\hline
\end{tabular}

射线结构分析表明，这些配合物都是中性席夫碱镱氯化 物(Scheme 1). 本文改用席夫碱锂盐取代钠盐进行反应 时, 首次得到离子对席夫碱镱锂氯化物 $\mathrm{YbLCl}_{3} \mathrm{Li}-$ $(\mathrm{DME})_{3}(\mathbf{1})(\mathrm{Eq} .1)$. 配合物 1 经元素分析和 IR 光谱表征, 元素分析结果与目标配合物组成基本一致; 红外光谱中 $1611 \mathrm{~cm}^{-1}$ 处强吸收可归于 $\mathrm{C}=\mathrm{N}$ 键的伸缩振动. 由 $\mathrm{X}$ 射线单晶衍射进一步确认了其分子结构. 配合物 1 易溶 于 THF 和 DME 中, 在甲苯中有一定溶解性, 难溶于己 烷. 我们试图在甲苯中培养单晶没有成功, 可能是甲苯 的配位能力比较弱. 当用 $\mathrm{THF} /$ 正己烷的混合溶剂培养 晶体时, 得到的晶体离开溶剂时, 很快就脱溶剂开裂, 没法测试. 另外, 把 $\mathrm{YbCl}_{3}$ 换成其它稀土氯化物时, 反 应现象基本一致, 遗憾的是, 到目前还没有得到良好质 量的单晶.

\section{2 席夫碱上酚羟基的质解反应}

在消除席夫碱上羟基质子的方法上，我们课题组做 了许多有益的工作. 在参考文献的基础上, 我们考察过
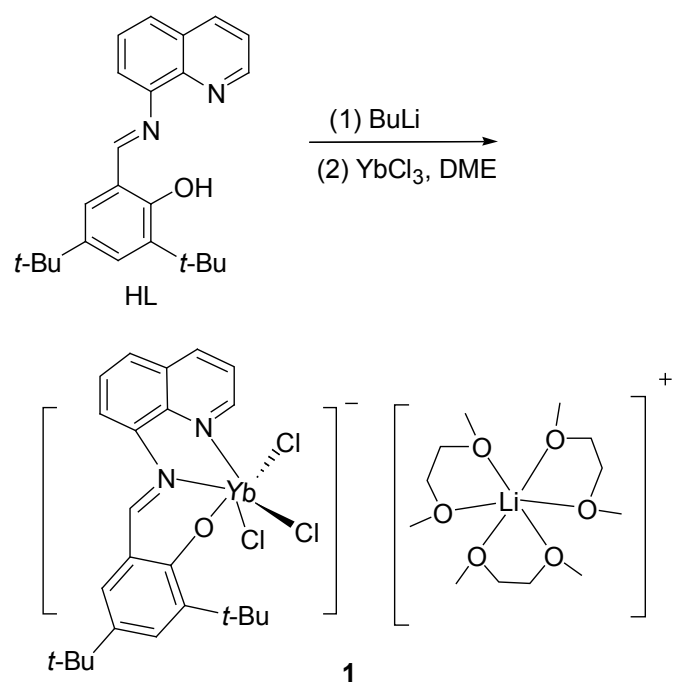

用金属烷基化合物(如丁基锂)、氢化物(如氢化钠)、活 泼金属(如钠)、金属胺化物(如硅胺稀土)、金属芳氧(烷 氧)化物(如三芳氧稀土配合物)等来反应掉该质子.

我们用丁基锂去质子，当控制好丁基锂的用量和反 应温度时，可以得到正常席夫碱锂盐，用它原位和稀土 氯化物反应，可得到正常的席夫碱稀土氯化物(Eq. 1). 如果反应温度和试剂用量控制不好时，我们发现，可能 在中和掉羟基质子的同时，出现加成 $\mathrm{C}=\mathrm{N}$ 双键现象 (Scheme 2) ${ }^{[13]}$ ，文献也曾报道用稀土烷基化合物和席夫 碱进行质解反应时，往往会发生烷基加成亚胺键的现 象 $^{[14]}$. 用锂盐与稀土氯化物反应时, 由于氯化锂在有机 溶剂中溶解性比较大，较难除去，所以在结晶产物中往 往会夹杂锂盐，形成离子型配合物(Eq. 1).

而用氢化钠去席夫碱配体上羟基质子时，不会出 现还原 $\mathrm{C}=\mathrm{N}$ 键的现象. 与席夫碱反应生成的钠盐再和 稀土氯化物反应时, 由于氯化钠在有机溶剂中溶解性比 较小，容易除去，所以在结晶产物中一般不会夹杂钠盐， 能得到中性配合物 ${ }^{[9]}$.

我们曾试图用活泼金属钠来消除席夫碱上羟基质 子. 当把金属钠加入到席夫碱 $\mathrm{HL}^{1}\left[\mathrm{HL}^{1}=2-\mathrm{HOC}_{6} \mathrm{H}_{4}-\right.$ $\mathrm{CH}=\mathrm{N}-2,6-(i-\mathrm{Pr})_{2} \mathrm{C}_{6} \mathrm{H}_{3}$ 的 $\mathrm{THF}$ 溶液中反应，然后在 $\mathrm{DME}$ 溶剂中结晶, 得到席夫碱配体被还原的钠盐 $\left(\mathrm{NaL}{ }^{1} \mathrm{DME}\right)_{2}$ (Eq. 2，图 1)，即钠在反应掉羟基质子的同 时, $\mathrm{CH}=\mathrm{N}$ 键也被还原成 $\mathrm{CH}_{2}-\mathrm{NH}$; 该化合物在 $\mathrm{THF}$ 中溶解性极好，能溶于甲苯和 DME 中，不溶于己烷. $\left(\mathrm{NaL}^{1}{ }^{1} \mathrm{DME}\right)_{2}$ 分析数据如下: IR (KBr) v: 3362 (s), 3051 (s), 2962 (s), 2886 (s), 1621 (s), 1589 (s), 1446 (s), 1384 (m), 1330 (m), 1280 (s), 1145 (s), 1062 (m), 1033, 856 (m), $840(\mathrm{~m}), 745(\mathrm{~s}), 588(\mathrm{~m}), 517(\mathrm{~m}) \mathrm{cm}^{-1}$. Anal. calcd for $\mathrm{C}_{46} \mathrm{H}_{66} \mathrm{~N}_{2} \mathrm{O}_{6} \mathrm{Na}_{2}$ : C 69.96, H 8.36, N 3.35, Na 5.83; found C 69.49, H 8.16, N 3.52, Na 5.74. 钠盐的晶体学数 据：单斜晶体, $P 2_{1} / a$ 空间群, 晶胞参数: $a=1.1475(5)$ 


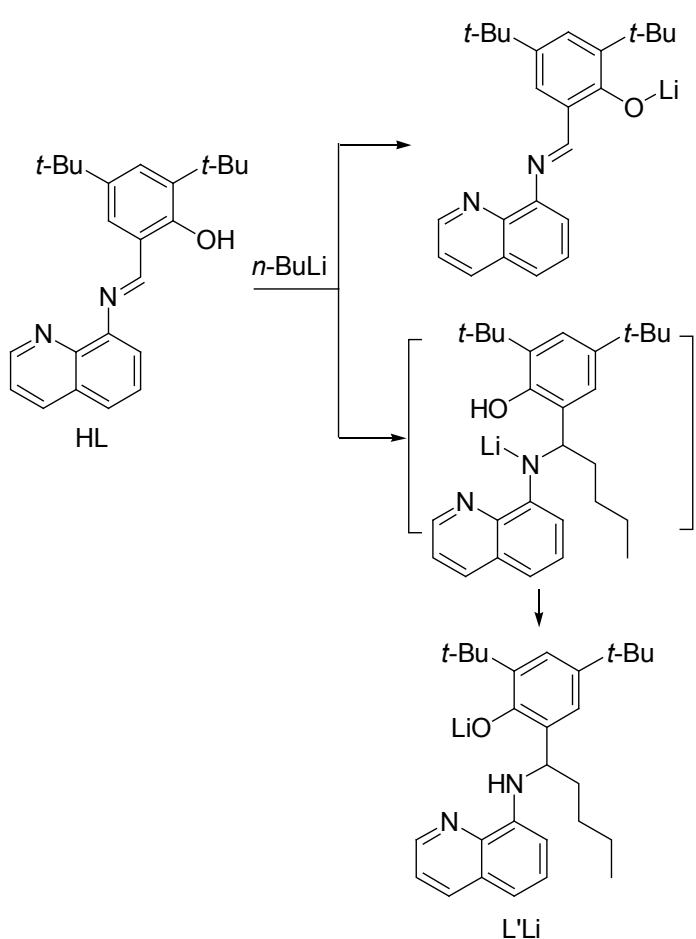

Scheme 2

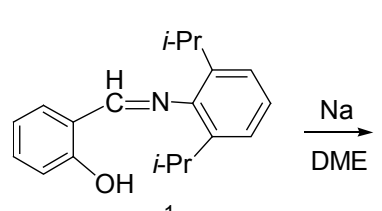

$\mathrm{HL}^{1}$

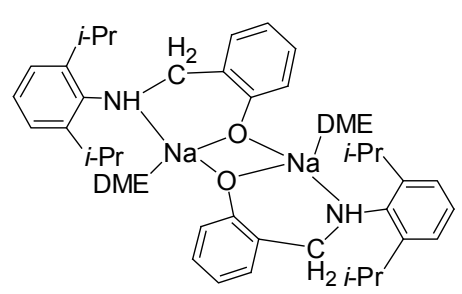

$\left(\mathrm{NaL}^{1} \mathrm{DME}\right)_{2}$

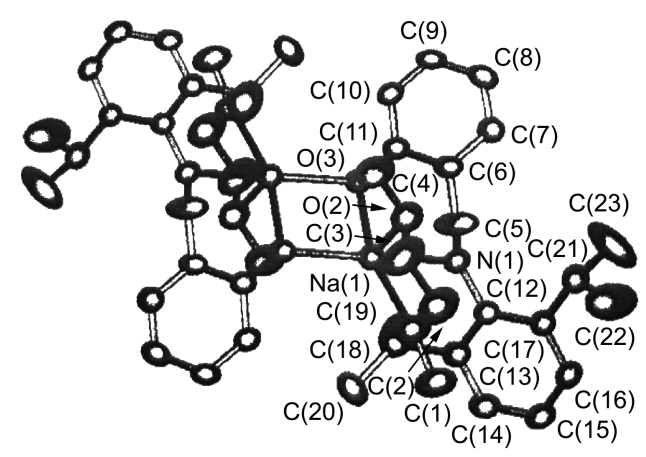

图 $1\left(\mathrm{NaL}^{1} \mathrm{DME}\right)_{2}$ 的结构图

Figure 1 ORTEP diagram of the complex $\left(\mathrm{NaL}^{1} \mathrm{DME}\right)_{2}$

$\mathrm{nm}, b=1.3987(5) \mathrm{nm}, c=1.4278(6) \mathrm{nm}, \alpha=90.00^{\circ}, \beta=$ $95.704(9)^{\circ}, V=2.2803(13) \mathrm{nm}^{3}, Z=4, F(000)=856, M_{\mathrm{r}}=$
789.04, $D_{\mathrm{c}}=1.152 \mathrm{~g} \bullet \mathrm{cm}^{-3}, R_{1}=0.069, w R_{2}=0.081$. 主要 键长和键角: $\mathrm{Na}(1)-\mathrm{O}(1) 0.2466(19) \mathrm{nm} ; \mathrm{Na}(1)-\mathrm{O}(2)$ $0.2347(2) \mathrm{nm}, \mathrm{Na}(1)-\mathrm{O}(3) 0.2283(18) \mathrm{nm}, \mathrm{Na}(1)-\mathrm{O}(3)^{*}$ $0.2250(18) \mathrm{nm}, \mathrm{Na}(1)-\mathrm{N}(1) \quad 0.2479(2) \mathrm{nm}, \mathrm{O}(1)-$ $\mathrm{Na}(1)-\mathrm{O}(2)=71.66(7)^{\circ}, \mathrm{O}(2)-\mathrm{Na}(1)-\mathrm{O}(3) 92.86(7)^{\circ}$, $\mathrm{O}(3)-\mathrm{Na}(1)-\mathrm{O}(3)^{*} 88.83(7)^{\circ}, \mathrm{O}(3)^{*}-\mathrm{Na}(1)-\mathrm{N}(1)$ $79.99(6)^{\circ}, \mathrm{Na}(1)-\mathrm{O}(3)-\mathrm{Na}(1)^{*} 91.17(7)^{\circ}$.

用硅胺金属化合物中和席夫碱差基质子时(Scheme $1)$, 在低温时可以获得正常席夫碱稀土胺化物 $\mathrm{YbL}_{2}[\mathrm{~N}(\mathrm{TMS})]$, 而较高温度时, 得到 $\mathrm{C}=\mathrm{N}$ 还原偶联配 合物 $\mathrm{YbL}$ ", 即硅胺负离子不仅中和了羟基质子，而且消 除了其中一个喹啉环上的 $\mathrm{C}-\mathrm{H}$ 质子, 该 $\mathrm{C}$ 与被还原的 $\mathrm{C}=\mathrm{N}$ 中的 $\mathrm{C}$ 形成一个新的 $\mathrm{C}-\mathrm{C}$ 单键, 从而得到一个

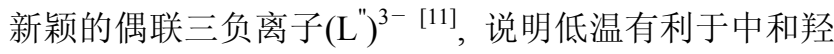
基质子。用 (THF) $\operatorname{LiLn}\left(\mathrm{N}-i-\mathrm{Pr}_{2}\right)_{4}$ 来消除类似席夫碱 $\mathrm{HL}^{2}$ (吡啶胺水杨醛席夫碱)上羟基质子时(Scheme 3), 在 室温下得到的是非正常席夫碱稀土胺化物: LiLn(THF)$\left[3,5-(t-\mathrm{Bu})_{2}-2-\mathrm{OC}_{6} \mathrm{H}_{2} \mathrm{CH}\left(\mathrm{N}-i-\mathrm{Pr}_{2}\right) \mathrm{NC}_{5} \mathrm{H}_{4} \mathrm{~N}\right]_{2}$, 即二异丙基 胺基向两个席夫碱上的 $\mathrm{C}=\mathrm{N}$ 发生了迁移，原来的三齿 配体在 $\mathrm{C}=\mathrm{N}$ 双键被加成后变成了四齿胺基芳氧基配 体. 而改用体积较大的硅胺稀土 $\left[\mathrm{N}(\mathrm{TMS})_{2}\right]_{3} \operatorname{Ln}(\mu-\mathrm{Cl})-$ $\mathrm{Li}(\mathrm{THF})_{3}$ 时, 就得到了正常席夫碱稀土胺化物 $\mathrm{LnL}_{2}^{2}\left[\mathrm{~N}(\mathrm{TMS})_{2}\right]$, 说明氨基体积大可以避免氨基对 $\mathrm{C}=$ $\mathrm{N}$ 键的插入反应，从而得到正常配合物 ${ }^{[15]}$.

用金属芳氧化物与席夫碱进行交换反应时，不会出 现还原偶联等异常现象(Scheme 1), 如 $\mathrm{HL}+\mathrm{Yb}(\mathrm{OAr})_{3}=$ $\mathrm{YbL}(\mathrm{OAr})_{2}+\mathrm{HOAr}^{[9]}$.

\section{3 配合物 1 晶体结构分析}

图 2 是配合物 1 的分子结构图. 表 2 列出了其主要 的键长、键角数据. 由图可知, 目标配合物是由阴离子 $\left[\mathrm{YbLCl}_{3}\right]^{-}$和阳离子 $\left[\mathrm{Li}(\mathrm{DME})_{3}\right]^{+}$两部分组成的离子对化 合物. 在阴离子部分, 中心三价镱离子与三个氯离子、 一个席夫碱的三个原子 $(\mathrm{O}, \mathrm{N}, \mathrm{N})$ 键合, 配位数为六, 呈 稍微扭曲的八面体构型. $\mathrm{O}(1), \mathrm{N}(1), \mathrm{N}(2)$ 和 $\mathrm{Cl}(2)$ 形成一 个平面四边形, 该四边形的四个内角和为 $360^{\circ}[\mathrm{O}(1)-$ $\mathrm{Yb}(1)-\mathrm{N}(1)=77.5(2)^{\circ}, \mathrm{N}(1)-\mathrm{Yb}(1)-\mathrm{N}(2)=68.4(2)^{\circ}$, $\mathrm{N}(2)-\mathrm{Yb}(1)-\mathrm{Cl}(2)=93.52(19)^{\circ}, \mathrm{O}(1)-\mathrm{Yb}(1)-\mathrm{Cl}(2)=$ $\left.120.84(18)^{\circ}\right], \mathrm{Cl}(1)$ 和 $\mathrm{Cl}(3)$ 原子占据八面体轴向位置, 键 角 $\mathrm{Cl}(1)-\mathrm{Yb}(1)-\mathrm{Cl}(3)=173.43(12)^{\circ}$, 接近一个平角. $\mathrm{Yb}-\mathrm{O}$ 键长为 $0.2101(5) \mathrm{nm}$, 它和文献报道的相应键长 相当: $\left[\mathrm{YbLCl}_{2}(\mathrm{DME})\right], 0.2115(2) \mathrm{nm}$; $\left[\mathrm{YbL}\left(\mathrm{CH}_{3} \mathrm{C}_{5} \mathrm{H}_{4}\right) \mathrm{Cl}\right.$ (THF)], 0.2116(3) nm; [YbL(OAr) $)_{2}, 0.2104(2) \mathrm{nm}^{[9]}$; $\left[\left\{2-\mathrm{OC}_{6} \mathrm{H}_{4} \mathrm{CH}=\left(2,6-i-\mathrm{Pr}_{2} \mathrm{C}_{6} \mathrm{H}_{3}\right)\right\}_{2} \mathrm{YbCl}(\mathrm{THF})\right], 0.2110(7)$ 和 $0.210(1) \mathrm{nm}^{[16]} ;\left[\left\{\left(\eta^{5}-\mathrm{C}_{5} \mathrm{H}_{5}\right) \mathrm{Yb}-\left(\mu-\mathrm{OC}_{20} \mathrm{H}_{20} \mathrm{~N}_{2} \mathrm{O}\right)\right\}_{2}-\right.$ $\left(\mu\right.$-THF)(THF)], 0.215(2) $\mathrm{nm}^{[17]} ; \mathrm{L}_{2} \mathrm{YbCl}, 0.2146(6) \mathrm{nm}$ 和 


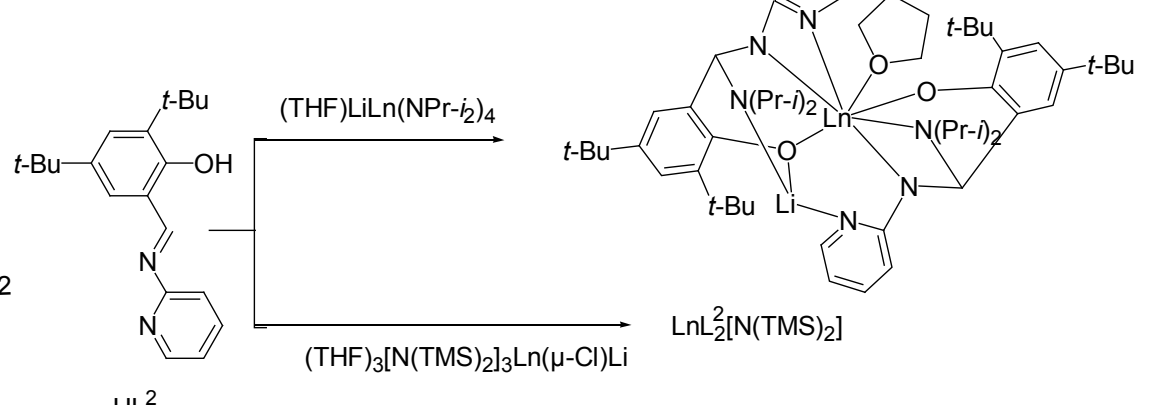

$\mathrm{HL}^{2}$

Scheme 3
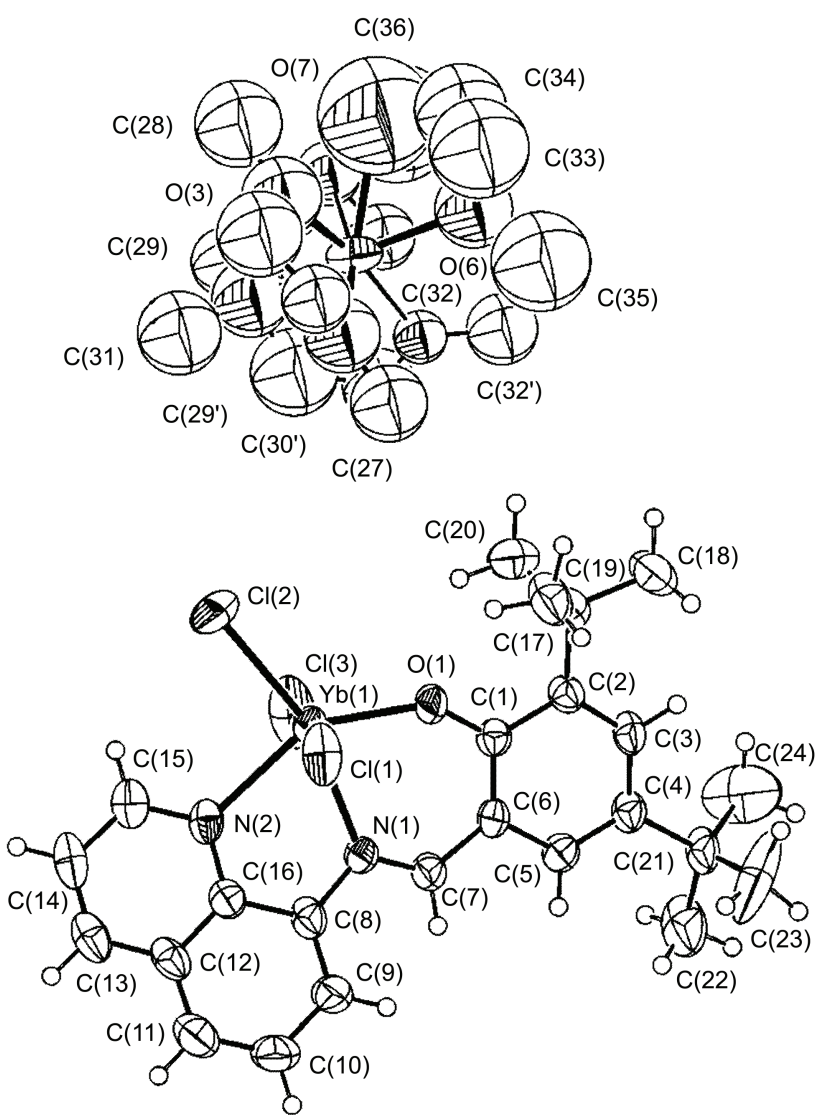

图 2 配合物 1 结构图

Figure 2 ORTEP diagram of the complex 1

$0.2144(6) \mathrm{nm}^{[13]} . \mathrm{Yb}-\mathrm{N}(\mathrm{C}=\mathrm{N})$ ) 键长是 $0.2389(7) \mathrm{nm}$, 它 和我们先前报道的配合物中相应键长一致: [YbLCl ${ }_{2}$ (DME)], 0.2386(3) nm; [YbL(OAr) 2 , 0.2359(3) $\mathrm{nm}^{[9]}$. $\mathrm{Yb}-\mathrm{N}$ (quinoline) 键长为 0.2403(7) nm, 它稍大于 $\mathrm{Yb}-\mathrm{N}(\mathrm{C}=\mathrm{N})$ 键长, 可能是由于喹啉环的刚性引起的. 三个 $\mathrm{Yb}-\mathrm{Cl}$ 键长接近, 分别为 $0.2551(3), 0.2566(3)$ 和 $0.2575(3) \mathrm{nm}$ (平均 $0.2564 \mathrm{~nm}$ ), 和前期报道的相应键长 相当: $\left[\mathrm{YbLCl}_{2}(\mathrm{DME}), 0.25934(8), 0.25654(9) \mathrm{nm}\right.$ (平均 $0.2579 \mathrm{~nm})$; [YbL $\left.\left(\mathrm{CH}_{3} \mathrm{C}_{5} \mathrm{H}_{4}\right) \mathrm{Cl}(\mathrm{THF})\right], 0.2594(1) \mathrm{nm}^{[9]}$;
但大于配合物 $\left[\left\{2-\mathrm{OC}_{6} \mathrm{H}_{4} \mathrm{CH}=\mathrm{N}\left(2,6-i-\mathrm{Pr}_{2} \mathrm{C}_{6} \mathrm{H}_{3}\right)\right\}_{2} \mathrm{YbCl}-\right.$ $(\mathrm{THF})]$ 中相应键长 $0.2497(2) \mathrm{nm}^{[16]}$.

表 2 配合物 1 的主要键长 $(\mathrm{nm})$ 和键角 $\left(^{\circ}\right)$

Table 2 Selected bond lengths $(\mathrm{nm})$ and angles $\left(^{\circ}\right)$ for complex 1

\begin{tabular}{llll}
\hline $\mathrm{Yb}(1)-\mathrm{O}(1)$ & $0.2101(5)$ & $\mathrm{O}(1)-\mathrm{Yb}(1)-\mathrm{N}(1)$ & $77.5(2)$ \\
$\mathrm{Yb}(1)-\mathrm{N}(1)$ & $0.2389(7)$ & $\mathrm{N}(1)-\mathrm{Yb}(1)-\mathrm{N}(2)$ & $68.4(2)$ \\
$\mathrm{Yb}(1)-\mathrm{N}(2)$ & $0.2403(7)$ & $\mathrm{N}(2)-\mathrm{Yb}(1)-\mathrm{Cl}(2)$ & $93.52(19)$ \\
$\mathrm{Yb}(1)-\mathrm{Cl}(1)$ & $0.2566(3)$ & $\mathrm{O}(1)-\mathrm{Yb}(1)-\mathrm{Cl}(2)$ & $120.84(18)$ \\
$\mathrm{Yb}(1)-\mathrm{Cl}(2)$ & $0.2551(3)$ & $\mathrm{Cl}(1)-\mathrm{Yb}(1)-\mathrm{Cl}(3)$ & $173.43(12)$ \\
$\mathrm{Yb}(1)-\mathrm{Cl}(3)$ & $0.2575(3)$ & $\mathrm{O}(3)-\mathrm{Li}(1)-\mathrm{O}(2)$ & $73.2(9)$ \\
$\mathrm{O}(2)-\mathrm{Li}(1)$ & $0.218(3)$ & $\mathrm{O}(4)-\mathrm{Li}(1)-\mathrm{O}(5)$ & $72.1(11)$ \\
$\mathrm{O}(3)-\mathrm{Li}(1)$ & $0.213(3)$ & $\mathrm{O}(6)-\mathrm{Li}(1)-\mathrm{O}(7)$ & $61.4(13)$ \\
$\mathrm{O}(4)-\mathrm{Li}(1)$ & $0.217(3)$ & & \\
$\mathrm{O}(5)-\mathrm{Li}(1)$ & $0.221(3)$ & & \\
$\mathrm{O}(6)-\mathrm{Li}(1)$ & $0.224(3)$ & & \\
\hline
\end{tabular}

\section{References}

[1] Emslie, D. J. H.; Piers, W. E.; Parvez, M.; McDonald, R. Organometallics 2002, 21, 4226.

[2] Lauterwasser, F.; Hayes, P. G.; Bróse, S.; Piers, W. E.; Schafer, L. L. Organometallics 2004, 23, 2234.

[3] Evans, W. J.; Fujimoto, C. H.; Ziller, J. W. Chem. Commun. 1999, 311.

[4] Evans, W. J.; Fujimoto, C. H.; Ziller, J. W. Polyhedron 2002, 21, 168.

[5] Blech, P.; Floriani, C.; Chiesi-Villa, A.; Guastini, C. J. Chem. Soc., Dalton Trans. 1990, 3557.

[6] Yousaf, M.; Liu, Q.-C.; Huang, J.-L.; Qian, Y.-L.; Chan, A. S.-C. Inorg. Chem. Commun. 2000, 3, 105.

[7] Li, B.-Y.; Yao, Y.-M.; Wang, Y.-R.; Zhang, Y.; Shen, Q. J. Rare Earths 2008, 26(4), 469.

[8] Li, B.-Y.; Yao, Y.-M.; Wang, Y.-R.; Zhang, Y.; Shen, Q. Inorg Chem. Commun. 2008, 11(3), 349.

[9] Li, B.-Y.; Yao, Y.-M.; Wang, Y.-R.; Zhang, Y.; Shen, Q. Polyhedron 2008, 27(2), 709.

[10] Li, B.-Y.; Yao, Y.-M.; Wang, Y. R.; Zhang, Y.; Shen, Q. J Organomet. Chem. 2009, 694(15), 2409.

[11] Han, F.-B.; Li, B.-Y.; Zhang, Y.; Wang, Y.-R.; Shen, Q. Organometallics 2010, 29, 3467.

[12] Li, B.-Y.; Yao, Y.-M.; Wang, Y.-R.; Zhang, Y.; Shen, Q. Chin. J. Org. Chem. 2010, 30(11), 1711 (in Chinese). 
(李邦玉, 姚英明, 王耀荣, 张勇, 沈其, 有机化学, 2010, 30(11), 1711.)

[13] Li, B.-Y.; Yao, Y.-M.; Wang, Y.-R.; Zhang, Y.; Shen, Q. Polyhedron 2011, 30, 207.

[14] Cameron, P. A.; Gibson, V. C.; Redshaw, C.; Segal, J. A.; White, A. J. P. J. Chem. Soc., Dalton Trans. 2002, 415.
[15] Qin, D.-W.; Han, F.-B.; Yao, Y.-M.; Zhang, Y.; Shen, Q. J. Chem. Soc., Dalton Trans., 2009, 5535.

[16] Yu, L.-B.; Yao, Y.-M.; Shen, Q.; Zhang, J.; Wu, L. X.; Ye, L. Chin. J. Chem. 2003, 21(4), 442.

[17] Liu, Q.-C.; Ding, M.-X. J. Organomet. Chem. 1998, 553(1), 179.

(Zhao, X.; Lu, Z.) 